\title{
Dynamic Self-Organizing Generation Algorithm of Complex Assembly Network Model
}

\author{
HOU Wenjun ${ }^{1,2, *}$, JIN Y $^{1,2}$, LI Tiemeng $^{1,2, *}$ and SUN Hanxu ${ }^{1}$ \\ ${ }^{1}$ Beijing University of Posts and Telecommunications, Beijing 100876, China \\ ${ }^{2}$ Beijing Key Laboratory of Network Systems and Network Culture, Beijing 100876, China
}

Received: 7 Apr. 2013, Revised: 11 Aug. 2013, Accepted: 13 Aug. 2013

Published online: 1 Nov. 2013

\begin{abstract}
In this paper, a model was proposed to describe the structure and the assembly mechanism of assembly network based on six principles: (1) the network has fixed number of vertices; (2) each vertex has limited degree; (3) the network shows clustering; (4) linking take place between pairs of vertices at a rate which is high if the degree limitation of this pair is large and the distance is close and low otherwise; (5) many sub-networks are growing in the same time and connected together at last; (6) there is relatively stable structure. Using computer simulations, the model which was proposed in this paper and incorporated all of these features can reproduce many of the features of real assembly network, including high level of clustering, the feature which is partially consistent with the scale-free network, and local-world growing.
\end{abstract}

Keywords: Complex network, assembly network, small world, scale-free, assembly mechanism.

\section{Introduction}

Many real-world systems can be represented by networks-nodes or vertices which are considered as components in systems connected together by links or edges which are considered as the connections between vertices. The classic example is the World-Wide Web. The vertices in this network are Web pages and the (directed) edges between them are hyperlinks from one page to another. In the last 10 years there has been a substantial amount of interest in network structure and function within the physics community $[1,2,3,4]$. In particular, it turns out that many of the techniques of statistical physics, such as scaling and renormalization group methods, Monte Carlo simulation, and mean-field theory, are well suited to the study of these systems.

There are two pioneering work in the research on the feature [5] of complex network-the small-world feature proposed by Watts and in Strogatz 1998 and the scale-free feature [6] proposed by Barabasi and Albert in 1999. Besides, people found that there are community structures in some complex networks [7]. For example, a group people with similar background or hobbies may form an association or community. At present, the research on social network has become one hotspot in the field of complex network $[8,9,10,11]$. In other research, people found that connection mechanism used in scale-free network based on global optimization can only work in some local-world when individuals cannot see the overall situation. A local-world model proposed by LI Xiang and CHEN Guangrong [12] can be used to describe this well.

There is another type of network which has some of the features of both social network and local-world network. For example in the railway network, we consider cities as vertices, and then connection between two nearby cities will take place at a higher rate which is coincident with the features of local-world network model; the overall number of cities is stable and the degree of each city is limited which is coincident with the features of social network model. Besides, this type of network has some particular features. For example, the location of each city is fixed and known so that it is easy to calculate the distance between two cities; the structure of the railway network will be stable when consistent with some conditions. This situation is very similar to the assembly process of mechanical product. In the design stage, the number, the spatial location and some other information of parts have already been known. And during the assembly process, parts will prefer to make an assembly connection with their nearby specific parts. So

\footnotetext{
*Corresponding author e-mail: wenjunh2113@263.net, tiemeng2000@gmail.com
} 
analyzing the static characteristics of this type of network will help us to understand the structure of mechanical products; analyzing the growing mechanism of this type of network will provide theory foundation for realizing the automation in the mechanical product assembly. In this paper, we call this type of network assembly network.

In this research, the assembly (growing) mechanism is analyzed and described at first; then a model is made to simulate the structure and assembly mechanism of assembly network; at last, eight mechanical products are used to be analyzed as samples, and compared to the network generated by our model to demonstrate the effectiveness of our model. In the same spirit as some other successful models of complex networks, this model is based on simple stochastic processed and do not attempt to capture the microscopic details which have smaller effect to the whole system to make sure that the model is simple and easy enough to understand.

\section{Assembly mechanism of networks}

At present, there are two types of networks whose growing mechanism is representative, scale-free network and social network. The key elements in scale-free network are (1) continual addition of both vertices and edges to the network as time passes and (2) preferential attachment, which means that edges are more likely to connect to vertices of high degree than to ones of low degree. Meanwhile, the key elements in social network are (1) fixed number of vertices, (2) limited degree of each vertex, (3) high clustering and (4) decay of connections. There are some other elements which have the effect to growing mechanism of network, but the most important ones are the 6 elements mentioned above. In generally, the growing mechanism of most of other types of network can be measured by some of the 6 elements.

However, growing mechanism of these type are, as mentioned above, quite inappropriate as assembly mechanism of assembly network-for a number of reasons, as follows.

(1) New vertices are of course added to assembly network all the time. However, the timescale on adding and removing parts into assembly network is very long, which is very similar to the timescale on social network. For example in mechanical product network, the speed of adding and removing parts is higher in the initial stage of assembling products, but slower in the latter stage because the parts which is going to be added or removed are just to affect the appearance usually. For this reason, we expect that the addition and removal of vertices will not be a major factor determining the assembly mechanism of assembly network. Also like social network, each vertex in assembly network has a characteristic of power which is used to maintain the connections with other vertices, and the power is limited.

(2) In scale-free network, vertices with more connections to other vertices attract new ones at a higher rate regardless of the real distance between vertices; but in assembly network, the real distance between vertices should not be ignored. So the structure of assembly network is not only controlled by the degree of vertices, but also affected by the real distance between vertices.

(3) The growing process of either scale-free network or small-world network is a growth process of one network or several networks, one of which is a core network (usually with the most number of vertices and so much larger than other networks). In contrast, the growing process of assembly network is a growth process of several sub-networks with equal position. All of them will join together at last.

(4) The characteristics of scale-free network and small-world network are used to evaluate the instantaneous structure of a network. The characteristics of social network are used to evaluate the dynamic structure of a network, and the structure of social network itself is of course changing all the time: people travel around making new friends and leaving old ones. Assembly network is a dynamic network, but there is relatively stable structure. Once this stable structure is established, it is very hard to change.

Taking each of these points into account, we propose the following items as a minimal set of features that a model of assembly network evolution should have.

(1) The network has fixed number of vertices;

(2) Each vertex has limited degree;

(3) The network shows clustering;

(4) Linking take place between pairs of vertices at a rate which is high if the degree limitation of this pair is large and the distance is close and low otherwise;

(5) Many networks are growing in the same time and connected together at last;

(6) There is relatively stable structure.

In the following sections we propose and study a model which has these properties to describe the assembly network.

\section{Model}

The most important mechanism in scale-free network is preferential attachment, which means how a newly added vertex is going to connect to other vertices in network. In contrast, the number of vertices $n$ in assembly network is fixed and the position of each vertex is fixed too. Every vertex is involved in network growing per unit time. All these vertices do not connect to other vertices randomly, but to the nearby vertices which have larger power and join together at last.

We propose the following mechanism for the growth of assembly network: pairs of individuals meet with a probability per unit time which depends on the distance between them, the total power and the residual power they have respectively. In the particular case of railway network, railway take place between pairs of cities at a rate which is low if the distance between this pair is long 
which may lead to high cost and high otherwise; in the mean while, a city prefer to connect to other cities which are more flourishing; but there is a recurring cost in terms of time and money to maintain a railway. So with limited resources cities can only maintain a certain number of them.

The distance $d i s_{i j}$ between given two vertices, $i$ and $j$, can be calculated by using their positions in space. Each vertex has limited power pow $_{i}$ to maintain its connections to other vertices. Obviously, the relationship between the current degree of a vertex $d_{i}$ and its total power pow $_{i}$ is: $d_{i} \leq$ pow $_{i}$. The difference between pow $w_{i}$ and $d_{i}$ is the residual power of the vertex $r_{i}$.

If only these mechanisms were in place, we would get a network which would grow until pow $w_{i}$ and $d_{i}$ of all or most vertices has the relationship of $p o w_{i}=d_{i}$, and then stop growing. The structure of the network would not change after its initial formation. In fact, Watts [13] has described just such a model on his research on social network, his -model, in which a hard upper limit is placed on the number of acquaintances an individual can have, and the model does indeed stop evolving once everyone has reached this limit. In the real world, however, assembly network do not stop growing so soon. It is hard to break a connection between two vertices; but as network growing, the connections will be broken at a rate when the structure of network is unable to meet some certain optimal condition. It is like during the developing of cities, the railway cannot be broken easily until a new railway which is shorter than the previous one between these cities has been built. It can be interpreted as the followings: assembly network grows with a decreasing speed and will stable at a relatively optimize structure. So in our model there are three steps per unit time: building connections between pairs of vertices, evaluating the structure of assembly network, breaking the connections which may reduce the efficiency of the network.

The probability per unit time $p_{i j}$ of a given two vertices, $i$ and $j$, meeting depends on three factors: (1) the total power pow $w_{i}$ and pow $w_{j}$ each vertex has, (2) the residual power $r_{i}$ and $r_{j}$ each vertex has and (3) the distance $d i s_{i j}$ between these two vertices, which is different from the distance in small-world network because here it means the real distance. We represent these factors by functions $f_{1}, f_{2}$ and $f_{3}$.

$$
p_{i j}=f_{1}\left(\text { pow }_{i}\right) f_{1}\left(\text { pow }_{j}\right) f_{2}\left(r_{i}\right) f_{2}\left(r_{j}\right) f_{3}\left(\text { dis }_{i j}\right)
$$

The function $f_{1}$ (pow) is a monotonically increasing function of pow. If there is a connection between vertex $i$ and vertex $j$, and pow $_{i}=\operatorname{pow}_{j}=1$, then these two vertices have no residual power to connect to other vertices. Therefore, the function $f_{1}$ (pow) should be very small when pow $=1$. But if $f_{1}($ pow $)=0$, then this vertex may has no connection to other vertices. So the range of function value of $f_{1}$ (pow) is $0<f_{1}$ (pow) $<1$. One possible functional form with these properties is like the following:

$$
f_{1}(\text { pow })=\frac{\arctan (\alpha *(\text { pow }-\beta))}{\pi}+\frac{1}{2}(\text { pow } \geq 1)
$$

and we have used this form for the simulations described here. The parameter $\alpha$ controls the slope rate of the function curve. The parameter $\beta$ is a transition value.

Like $f_{1}($ pow $)$, the function $f_{2}(r)$ is a monotonically increasing function too, where $r \leq$ pow. To avoid premature convergence of the structure of network, $r$ could be a negative and in the mean time the function $f_{2}(r)$ would be very small. According to these, we use a functional form like $f_{1}$ (pow) for the simulations:

$$
f_{2}(r)=\frac{\arctan (\gamma *(r-p o w))}{\pi}+\frac{1}{2}(r \leq \text { pow })
$$

where the parameter $\gamma$ controls the slope rate of the function curve.

The function $f_{3}\left(d i s_{i j}\right)$ is a monotonically decreasing function of $d i s_{i j}$. It means the longer the distance between a pair of vertices is, the smaller the function $f_{3}\left(d i s_{i j}\right)$ is. We use the following functional form for the simulations:

$$
f_{3}(\text { dis })=e^{-\delta * d i s}(\text { dis }>0)
$$

where the parameter $\delta$ controls the slope rate of the function curve.

The forms of $f_{1}, f_{2}$ and $f_{3}$ chosen here are somewhat ad hoc, but we have experimented with other forms and found the qualitative predictions of the model the same.

In the simulation for assembly network with this model, a normal assembly network should meet a few requirements: (1) the degree of each vertex should not be more than its total power, (2) the distance between a pair of vertices should be as short as possible, (3) the graph of the network should be fully connected and (4) the network is considered as stable when the structure of the network is nearly unchanged. According to these four requirements, we give each connection between a pair of vertices a score after evaluating the structure of assembly network. The value of the score depends on the distance between this pair of vertices, the power each vertex has, and the changing of the average distance of the whole network if this connection is removed. We use the functions $f_{1}$ and $f_{3}$ to represent the factors about the distance and the power, such as in the above functions. So the score of each connection can be calculated by the following function.

$$
s_{i j}=f_{3}\left(\text { dis }_{i j}\right) f_{1}\left(\text { pow }_{i}\right) f_{1}\left(\text { pow }_{j}\right) g\left(\langle l\rangle-\langle l\rangle^{\prime}\right)
$$

In the function $g\left(<l>-<l>^{\prime}\right),<l>$ represents the average distance of the network before removing this connection, and $\langle l\rangle$ represents the average distance of the network after removing this connection. The function $\left.g(<l>-<l\rangle^{\prime}\right)$ is larger when $\langle l>-<l\rangle^{\prime}>0$, which means the average distance is shortened after removing this connection, and smaller otherwise. The range of function value of $\left.g(\langle l\rangle-<l\rangle^{\prime}\right)$ is 


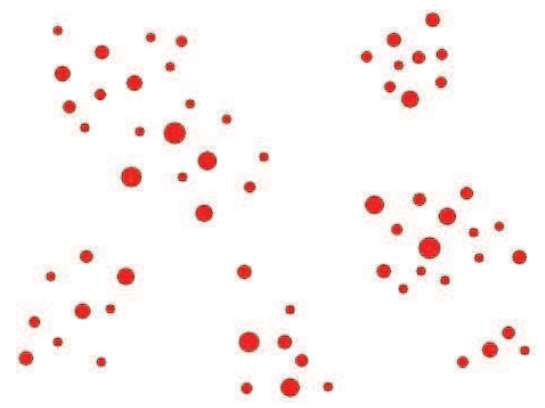

Fig. 1: Initialization of sample assembly network

$0<g\left(<l>-<l>^{\prime}\right)<1$, so one possible functional form with these properties is like the following.

$$
g\left(\langle l\rangle-\langle l\rangle^{\prime}\right)=\frac{\operatorname{arccot}\left(\langle l\rangle-\langle l\rangle^{\prime}\right)}{\pi}
$$

The power of each vertex is limited. So during the process of evaluating the network, if the degree of a vertex is larger than its power, then its one or several connections to other vertices should be removed. We give each connection to this vertex a score with equation (5) and rank all the scores, and then remove several connections with lower score based on the actual situation.

\section{Simulations}

There are two very important characteristics to evaluate complex network: small-world characteristic which is used to measure the level of clustering and scale-free characteristic which is used to describe the growing mechanism. Therefore, in our research we analyzed the network generated by our model and the mechanical products network based on above two characteristics, and then compared them.

\subsection{Simulation for our model}

Simulations can be initialized in a variety, so before analyzing the assembly mechanism of assembly network, we should discuss how to initialize the vertices in the network first. Assembly network shows high level of local clustering. If the vertices in network are located completely at random, then after growing up the network would be probably a random graph with low level of clustering. Therefore, in our simulation we use a sample network to test our model, as shown in Fig. 1. Obviously, the vertices in network can be easily divided into several communities.

In this simulation, we set $\alpha=\beta=1.0, \gamma=10.0$, $\delta=0.25$. Fig. 2 shows 3 snapshots of the network and the

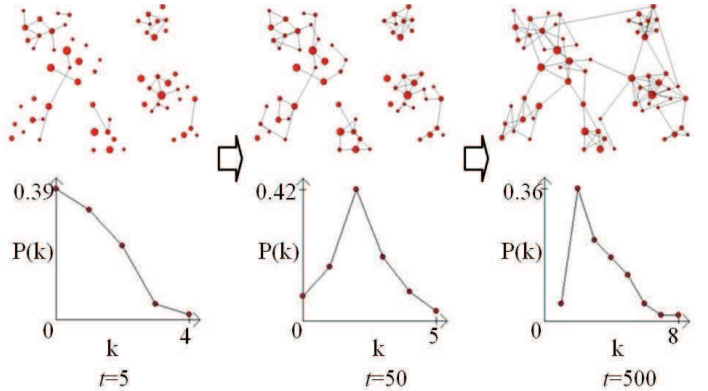

Fig. 2: Sample assembly network generated by our model and its degree distribution

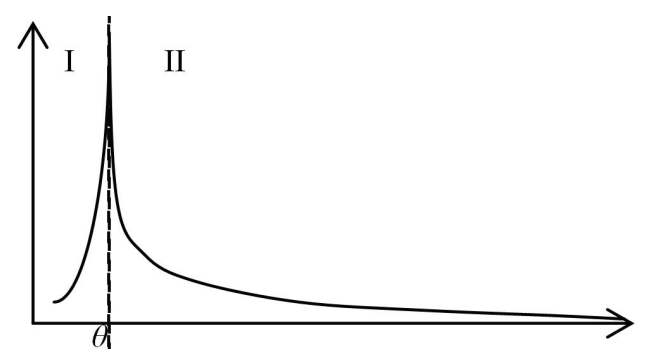

Fig. 3: A sketch map of the degree distribution of assembly network

corresponding degree distributions with $t=5, t=50$, and $t=500$ respectively, where $t$ represents the time since the network starting growing.

A scale-free network is a network whose degree distribution follows a power law, at least asymptotically $[6,14]$. That is, the smaller the vertices with same degree total, the more connections to other vertices the vertices have.

From Fig. 2, we can see that the vertices in the network prefer to connect their nearby vertices with higher power.

In the initial stage of the network growth, communities choose to grow separately and join together at last. It is a long time from $\mathrm{t}=50$ to $\mathrm{t}=500$, while the network does not have obvious changes. And in our simulation, after $t=500$ the change of the network is not obvious, too. So we considered that the network is in a relatively stable state. According to the degree distributions shown in Fig. 2, it can be seen that the degree distribution of assembly network does not follow the power law exactly. In assembly network, there is a threshold of degree $\theta$. The vertices whose degree is lower than the threshold have fewer connections to other vertices, and their degree distribution follows an exponential law, as shown in Fig. 3.

We set the clustering coefficient and the average distance as functions of time. When $\mathrm{t}=500$, the snapshots of their function images are shown in Fig. 4.

In Albert and Barabasis research [3], they thought if the probability $\mathrm{C}$ that two neighbors of a given vertex will 


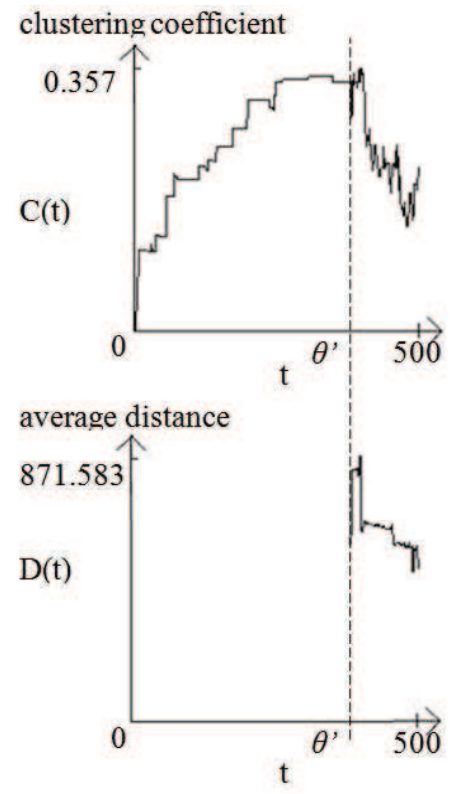

(a)

(b)

Fig. 4: Snapshots of the function images of the clustering coefficient and the average distance

be neighbors also of one another, also called the clustering coefficient, is greater by a factor of about five than in the corresponding baseline network, a random graph in which edges are assigned to vertices completely at random, and the network average distance is short enough, then we believe that the network has the characteristic of small-world. The average distance which is used to evaluate the characteristic of small-world network is measured as a function of the number of edges in the network, not the average value of all real distance between pairs of vertices.

When $t<\theta^{\prime}$, the average distance is infinite because the network is unconnected graph. Thus the clustering coefficient is far beyond our frame of reference.

When $t \geq \theta^{\prime}$, the network is connected graph. In the consideration of $C_{\text {random }} \approx 0.0525$ when $t=500$, we can get $C \gg C_{\text {random }}$ from Fig. 4(a), and the average network distance is declined from Fig. 4(b) at this time. And in our simulation, the topology of the network doesnt have significant changes when $t>500$. So we can conclude that the network generated by our model is up to the smallworld network.

We can see from Fig. 4(b) that the curve of the networks average distance isnt smoothly decreasing. The reasons are as follows.

(1) In the formula (5), the average distance is not the only factor of influencing whether one edge should be removed. Besides, we should also take the vertex power pow $_{i}$ and the distance $d i s_{i j}$ between different vertices into consideration.

(2) When evaluating the structure of assembly network, we calculate the scores of all the edges each

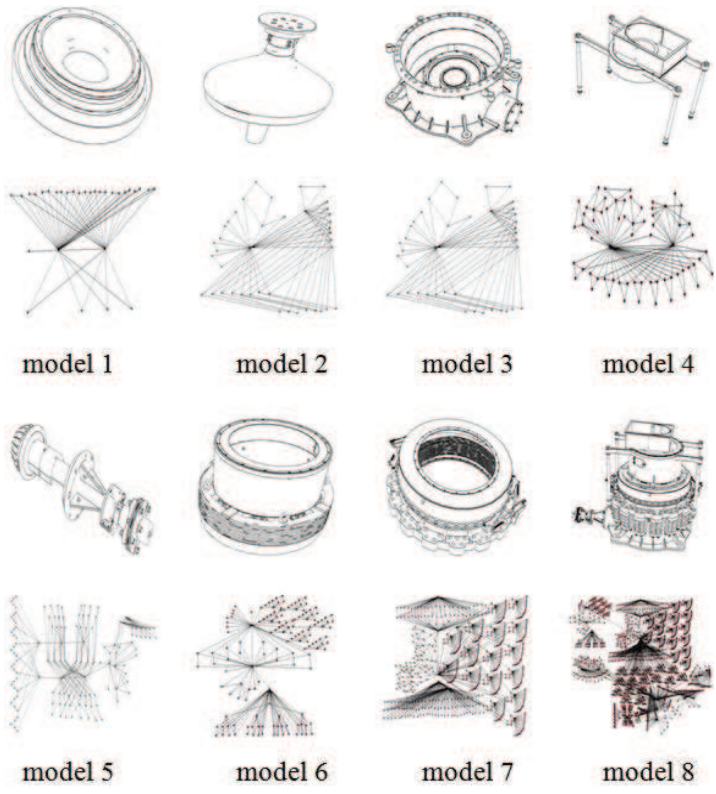

Fig. 5: 3D digital models and their corresponding assembly networks

time one vertex. So the evaluation is not an overall algorithm, and the average distance may get larger after removing some edges.

Even so, look from whole, the average distance of the network is declined.

\subsection{Analysis for mechanical products}

The part network of mechanical product is made up of parts which are considered as vertices and assembly relationships which are considered as edges between vertices. In this paper, statistical characteristics of assembly network are analyzed with these 8 mechanical products as the samples. The 3D digital models of these products and their corresponding part networks are shown in Fig. 5. Because the average distance which is used to evaluate the characteristic of small-world network is just measured as a function of the number of edges in the network, the networks shown in Fig. 5 are just 2D sketch maps and of course all vertices are not located on their real positions.

According to this, we analyzed the above 8 examples. The number of parts $n$, the number of assembly relationship $m$, the average distance $\langle l\rangle$, the clustering coefficient $C$, and the clustering coefficient $C_{\text {random }}$ of the corresponding baseline network are all shown in Table 1.

According to the number of parts and the average distance shown in Table 1, the average distance becomes longer with the increasing number of parts, which turns our nearly to be a logarithm relation, as shown in Fig. 6 . So we consider that the average distance of assembly 
Table 1: The parameters of assembly network of above 8 samples

\begin{tabular}{cccccc}
\hline index & $n$ & $m$ & $<l>$ & $C$ & $C_{\text {random }}$ \\
\hline model 1 & 31 & 58 & 1.8753 & 0.780 & 0.121 \\
model 2 & 41 & 72 & 2.4012 & 0.713 & 0.0856 \\
model 3 & 62 & 103 & 2.5304 & 0.364 & 0.0535 \\
model 4 & 67 & 124 & 2.7038 & 0.762 & 0.0552 \\
model 5 & 134 & 228 & 4.7166 & 0.473 & 0.0254 \\
model 6 & 154 & 272 & 4.3843 & 0.599 & 0.0229 \\
model 7 & 644 & 1140 & 5.4106 & 0.566 & 0.00550 \\
model 8 & 1442 & 2799 & 5.9497 & 0.581 & 0.00269 \\
\hline
\end{tabular}

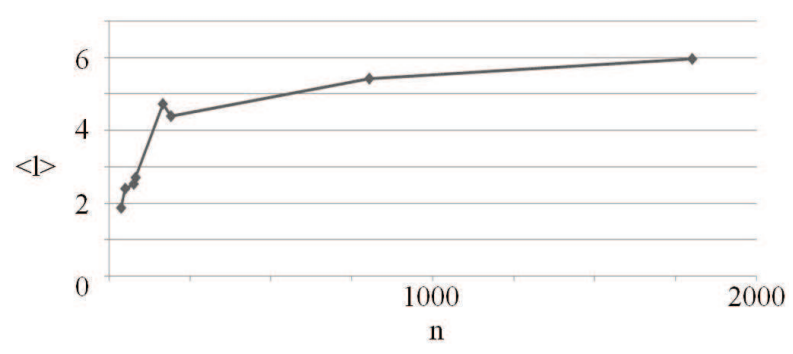

Fig. 6: Changes of the average distance $\langle l\rangle$ as a function of the number of parts $n$ in network

network is short. According to the clustering coefficient shown in Table 1 , we can also find that $C \gg C_{\text {random }}$. These two results show that assembly network has the characteristics of small-world network.

We calculated all the degree distributions of the above 8 examples and showed them in Fig. 7.

From the degree distributions of the above 8 examples in Fig. 7, we can see that the degree distribution of part network does not follow a power law exactly and its curve is similar to the functional curve of assembly network.

From the above, we can see that the network generated our model reproduces the six characters of assembly network we defined and its unique degree distribution, and the characters of small-world network. At the same time, the network is similar to real mechanical part network, so our model is workable.

\section{Conclusions}

In this paper, we proposed a new concept called assembly network which is used to represent the part network in mechanical products and the railway network between
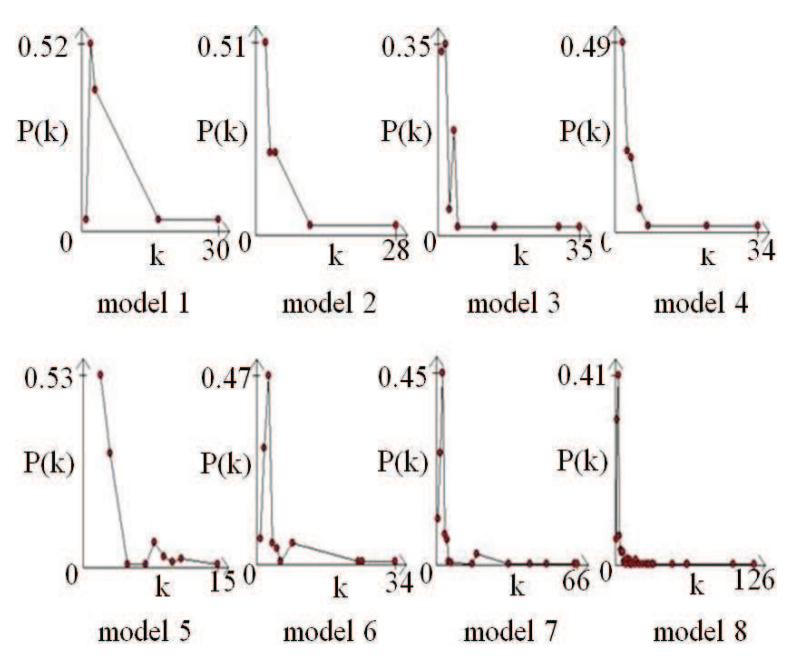

Fig. 7: Degree distributions of the above 8 samples

cities and some other similar networks. Assembly network is high level of clustering and partially consistent with the scale-free network. The crucial features which we find necessary to produce this type of network are six in number: (1) the network has fixed number of vertices; (2) each vertex has limited degree; (3) the network shows clustering; (4) linking take place between pairs of vertices at a rate which is high if the degree limitation of this pair is large and the distance is close and low otherwise; (5) many sub-networks are growing in the same time and connected together at last; (6) there is relatively stable structure. We proposed a model based on all these six features to make the simulation on assembly network. Simulation results show that the model of assembly network is efficient.

With the assembly network, we could describe the structure of mechanical product better, and it may be a feasible analysis tool for the assembly automation in the future. However, this paper represents only a first attempt at modeling and analyzing the evolution of the structure of assembly networks, and there are many possible directions for further study. For example, we have not proposed a strong appropriate description to the initialization of the assembly network, and a lot of parameters need to be customized by users. At the same time, the question of whether these are some other features besides the six features proposed in this paper is another problem to be studied.

\section{Acknowledgement}

This project is supported by National Natural Science Foundation of China (Grant No. 51075040), and Specialized Research Fund for the Doctoral Program Education (Grant NO. 20090005110014), and the Fundamental Research Funds for the Central Universities. 
The authors are grateful to the anonymous referee for a careful checking of the details and for helpful comments that improved this paper.

\section{References}

[1] NEWMAN M E J. Models of the small world[J]. Journal of Statistical Physics, 101, 819-841 (2000).

[2] STROGATZ S H. Exploring complex networks[J]. Nature, 410, 268-276 (2001).

[3] ALBERT R, BARABASI A L. Statistical mechanics of complex networks[J]. Reviews of modern physics, 74, 47-97 (2002).

[4] BOCCALETTI S, LATORAB V, MORENOD Y, et al. Complex networks structure and dynamics[J]. Physics Reports, 424, 175-308 (2006).

[5] WATTS D J, STROGATZ S H. Collective dynamics of 'small-world' networks[J]. Nature, 393, 440-442 (1998).

[6] BARABASI A L, ALBERT R. Emergence of scaling in random networks[J]. Science, 286, 509512 (1999).

[7] NEWMAN M E J. Detecting community structure in networks $[\mathrm{J}]$. The European Physical Journal BCondensed Matter, 38, 321-330 (2004).

[8] HOLME P, KIM B J. Growing scale-free networks with tunable clustering[J]. Physical Review E, 65, 026-107 (2002).

[9] JIN E M, GIRVAN M, NEWMAN M E J. Structure of growing social networks[J]. Physical Review E, 64, 046-132 (2001).

[10] NOH J D, JEONG H C, AHH Y Y, et al. Growing network model for community with group structure[J]. Physical Review E, 71, 036-131 (2005).

[11] XUAN Qi, LI Yanjun, WU Tiejun. Growth model for complex networks with hierarchical and modular structures[J]. Physical Review E, 73, 036-105 (2006).

[12] LI Xiang, CHEN Guanrong. A local-world evolving network model[J]. Physica A: Statistical Mechanics and its Applications, 328, 274-286 (2003).

[13] WATTS D J. Small worlds[M]. Princeton: Princeton University Press, (1999).

[14] Wikipedia. Scale-free network [EB/OL]. (2011-11-26) (2011-12-01)

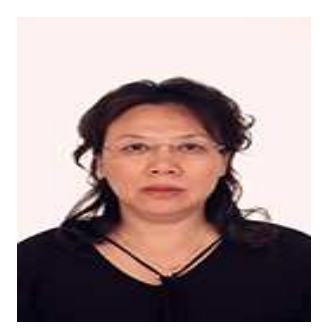

HOU Wenjun, born in 1963 , is currently a professor in Beijing University of Posts and Telecommunications, China. She received her $\mathrm{PhD}$ degree from Beijing University of Posts and Telecommunications, China, in 2010. Her research interests include virtual reality, intelligent assembly.

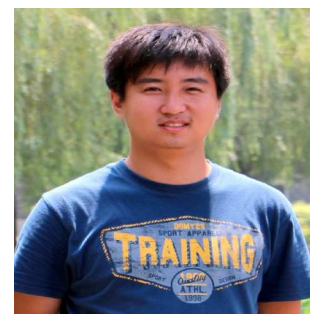

JIN Yu, born in 1984, is currently a $\mathrm{PhD}$ candidate in Automation School, Beijing University of Posts and Telecommunications, China. $\mathrm{He}$ received his bachelor degree from Huazhong University of Science and Technology, China, in 2007. His research interests include virtual reality, intelligent assembly.

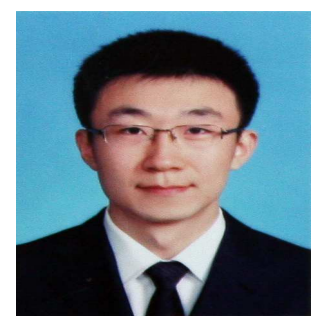

LI Tiemeng, born in 1984 , is currently a instructor in Beijing University of Posts and Telecommunications, China. He received his PhD degree from Beijing University of Posts and Telecommunications, China, in 2011. His research interests include context-aware computing, intelligent assembly. 